\title{
Sensitivity analysis explains quasi-one-dimensional current transport in two- dimensional materials
}

Boll, Mads; Lotz, Mikkel Rønne; Hansen, Ole; Wang, Fei; Kjær, Daniel; Bøggild, Peter; Petersen, Dirch Hjorth

Published in:

Physical Review B

Link to article, DOI:

10.1103/PhysRevB.90.245432

Publication date:

2014

Document Version

Publisher's PDF, also known as Version of record

Link back to DTU Orbit

Citation (APA):

Boll, M., Lotz, M. R., Hansen, O., Wang, F., Kjær, D., Bøggild, P., \& Petersen, D. H. (2014). Sensitivity analysis explains quasi-one-dimensional current transport in two-dimensional materials. Physical Review $B, 90(24)$, 245432. https://doi.org/10.1103/PhysRevB.90.245432

\section{General rights}

Copyright and moral rights for the publications made accessible in the public portal are retained by the authors and/or other copyright owners and it is a condition of accessing publications that users recognise and abide by the legal requirements associated with these rights.

- Users may download and print one copy of any publication from the public portal for the purpose of private study or research.

- You may not further distribute the material or use it for any profit-making activity or commercial gain

- You may freely distribute the URL identifying the publication in the public portal 


\title{
Sensitivity analysis explains quasi-one-dimensional current transport in two-dimensional materials
}

\author{
Mads Boll,,${ }^{1, *}$ Mikkel R. Lotz, ${ }^{1, *}$ Ole Hansen, ${ }^{2,3}$ Fei Wang, ${ }^{4}$ Daniel Kjær, ${ }^{2,5}$ Peter Bøggild, ${ }^{1}$ and Dirch H. Petersen ${ }^{1, \dagger}$ \\ ${ }^{1}$ Center for Nanostructured Graphene (CNG), Department of Micro- and Nanotechnology, Technical University of Denmark, \\ DTU Nanotech Building 345 East, DK-2800 Kgs. Lyngby, Denmark \\ ${ }^{2}$ Department of Micro- and Nanotechnology, Technical University of Denmark, DTU Nanotech Building 345 East, \\ DK-2800 Kgs. Lyngby, Denmark \\ ${ }^{3}$ Danish National Research Foundation's Center for Individual Nanoparticle Functionality (CINF), Technical University of Denmark, \\ DK-2800, Kgs. Lyngby, Denmark \\ ${ }^{4}$ Department of Electronic and Electrical Engineering, South University of Science and Technology of China, Shenzhen, China \\ ${ }^{5}$ CAPRES A/S, Scion-DTU, Building 373, DK-2800 Kgs. Lyngby, Denmark
}

(Received 3 October 2014; revised manuscript received 6 December 2014; published 29 December 2014)

\begin{abstract}
We demonstrate that the quasi-one-dimensional (1D) current transport, experimentally observed in graphene as measured by a collinear four-point probe in two electrode configurations $\mathrm{A}$ and $\mathrm{B}$, can be interpreted using the sensitivity functions of the two electrode configurations (configurations A and B represents different pairs of electrodes chosen for current sources and potential measurements). The measured sheet resistance in a four-point probe measurement is averaged over an area determined by the sensitivity function. For a two-dimensional conductor, the sensitivity functions for electrode configurations A and B are different. But when the current is forced to flow through a percolation network, e.g., graphene with high density of extended defects, the two sensitivity functions become identical. This is equivalent to a four-point measurement on a line resistor, hence quasi-1D transport. The sensitivity analysis presents a formal definition of quasi-1D current transport, which was recently observed experimentally in chemical-vapor-deposition graphene. Our numerical model for calculating sensitivity is verified by comparing the model to analytical calculations based on conformal mapping of a single extended defect.
\end{abstract}

DOI: 10.1103/PhysRevB.90.245432

PACS number(s): 02.60.Cb, 72.80.Vp, 73.23.-b

\section{INTRODUCTION}

The analysis and control of defects are ongoing topics for graphene films produced via chemical vapor deposition (CVD), which is the preferred method for producing cheap, high-quality graphene suited for large-scale integration. In graphene, such defects can be anything from lattice imperfections (grain boundaries) [1-3], physi- or chemisorbed adatoms, cracks [4], folds [5], areas with contamination, holes due to imperfect transfer from growth substrate, and surface corrugations responsible for various scattering effects reducing the carrier mobility and causing unintended variations in the current flow $[6,7]$.

In a recent study we experimentally observed quasi-onedimensional (1D) current transport in large area CVD graphene by micro-four-point-probe (M4PP) measurements [7]. We demonstrated how this could be qualitatively reproduced in a two-dimensional (2D) material with randomly distributed insulating line defects near the percolation threshold characterized by the filling factor $\rho \ell^{2}$, where $\rho$ was the defect density and $\ell$ the defect length [8]. This was done by inspecting the ratio of the two four-point resistances, $R_{\mathrm{A}} / R_{\mathrm{B}}$, measured by the electrode configurations shown in Fig. 1. For a material with uniform intrinsic transport properties, the resistance ratio solely depends on the sample geometry including electrode positions and takes on an ideal value of $\ln (4) / \ln (3)=1.262$ for a homogeneous 2D conductor, i.e., without any form of defects $[7,9]$. This was verified experimentally in a concurrent study

\footnotetext{
${ }^{*}$ These authors contributed equally to this work.

†dirch.petersen@nanotech.dtu.dk
}

for graphene without a high density of extended defects [9]. The notion of 1D-like transport or quasi-1D current transport represents the situation when the resistance ratio approaches 1 , i.e., the expected result for a $1 \mathrm{D}$ conductor or wire measurement $[7,8]$. For measurements on an inhomogeneous material, the sensitivity (or weighting function) of four-point resistance to small perturbations in the local transport properties has been studied both numerically [10-13] and analytically [14]. Similar studies have been conducted for finite point-like perturbations to include nonlinear effects on the sensitivity $[15,16]$, but the situation is different for highly nonuniform materials with extended insulating defects.

In this paper we present a numerical model of current flow in M4PP measurements in an initially 2D conducting sheet subjected to a large number of insulating line defects of random orientation. We show how the numerical model can be verified for simple situations, involving a single extended defect, using an analytical expression obtained via conformal mapping. The results are analyzed by mapping and comparing the sensitivity of measurements in different probe configurations. Through this technique, it is shown that the frequently occurring 1D signature is a result of the different probe configurations measuring the exact same area on the sample, due to defects confining the current. The approach can be expanded to a general framework for analyzing the sensitivity of other types of defects on electrical measurements.

\section{ANALYTICAL MODEL}

In a four-point probe measurement, a current $I$ is passed through the sample, using two of the four electrodes, while the resulting potential drop over the remaining two electrodes 


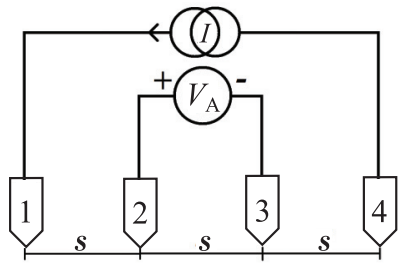

A

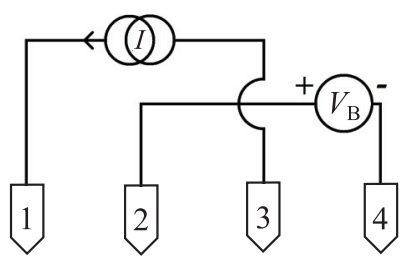

B

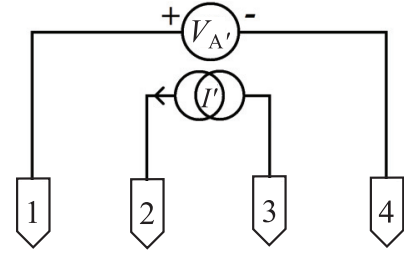

$\mathrm{A}^{\prime}$

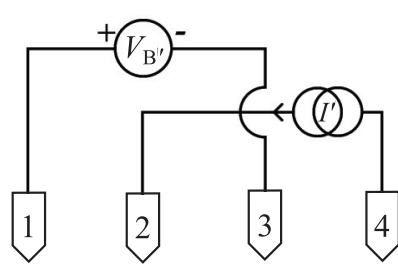

$\mathrm{B}^{\prime}$
FIG. 1. Schematic of four different probe configurations (A, B, $\mathrm{A}^{\prime}$ and $\mathrm{B}^{\prime}$ ). The probe pins are numbered 1 to 4 and are evenly spaced by a probe pitch $s$.

is measured. The measured resistance is determined by the ratio of measured voltage to applied current. The four-point resistances $R_{\mathrm{A}}, R_{\mathrm{B}}, R_{\mathrm{A}^{\prime}}$, and $R_{\mathrm{B}^{\prime}}$ are measured with the pin configurations (A, B, $\mathrm{A}^{\prime}$, and $\mathrm{B}^{\prime}$ ) defined in Fig. 1. Due to reciprocity, $R_{\mathrm{A}}=R_{\mathrm{A}^{\prime}}$ and $R_{\mathrm{B}}=R_{\mathrm{B}^{\prime}}$ in the absence of a magnetic field. To analytically model an M4PP measurement on a sample with a line defect, we turn to conformal mapping. Here a linear defect of finite length is mapped onto an infinite border, running along the $x$ axis. Thus the problem is reduced to that of a semi-infinite sheet, for which the electrostatic potential can be calculated for any probe position in the upper half-plane. In the mapping process, the probe pin coordinates are treated as complex numbers, $w=u+i v$ in real space and $z=x+i y$ in the conformal image plane, here $i=\sqrt{-1}$ is the imaginary unit. In the case of a short defect with length $\ell$, and its center at the origin of the coordinate system, the transformation of the pin coordinates from the $w$ plane to the $z$ plane and back is given by $z=i \sqrt{\frac{w-\ell / 2}{w+\ell / 2}}$ and $w=\frac{\ell}{2} \frac{1-z^{2}}{1+z^{2}}$, respectively. An example of this mapping procedure for three different M4PP pin positions around a defect is shown in Fig. 2.

The electrostatic potential $\Phi\left(\mathbf{r}, \mathbf{r}_{+}, \mathbf{r}_{-}\right)$at position $\mathbf{r}$ with two point current sources $\pm I$, at the positions $\mathbf{r}_{+}$and $\mathbf{r}_{-}$in the upper half-plane of the semi-infinite sheet, is found as a solution to the Laplace equation for an infinite sheet

$$
\Phi\left(\mathbf{r}, \mathbf{r}_{+}, \mathbf{r}_{-}\right)=\frac{I R_{0}}{2 \pi}\left(\ln \frac{\left|\mathbf{r}-\mathbf{r}_{-}\right|}{\left|\mathbf{r}-\mathbf{r}_{+}\right|}+\ln \frac{\left|\mathbf{r}-\overline{\mathbf{r}}_{-}\right|}{\left|\mathbf{r}-\overline{\mathbf{r}}_{+}\right|}\right),
$$

where $\overline{\mathbf{r}}_{ \pm}=\left(\bar{x}_{ \pm}, \bar{y}_{ \pm}\right)=\left(x_{ \pm},-y_{ \pm}\right)$are the positions of image current sources in the lower half-plane and $R_{0}$ is the sheet resistance. This image technique ensures that the current density $\mathbf{J}_{s}$ across the boundary between two half-planes is zero, $\mathbf{J}_{s} \cdot \mathbf{n}=0$, where $\mathbf{n}$ is the unit vector normal to the boundary, which is the correct boundary condition for the semi-infinite system. A detailed derivation of this potential is presented in Ref. [17]. The resistances are found using Ohm's law, for instance, $R_{\mathrm{A}}=\left[\Phi\left(\mathbf{r}_{2}, \mathbf{r}_{1}, \mathbf{r}_{4}\right)-\Phi\left(\mathbf{r}_{3}, \mathbf{r}_{1}, \mathbf{r}_{4}\right)\right] / I$.
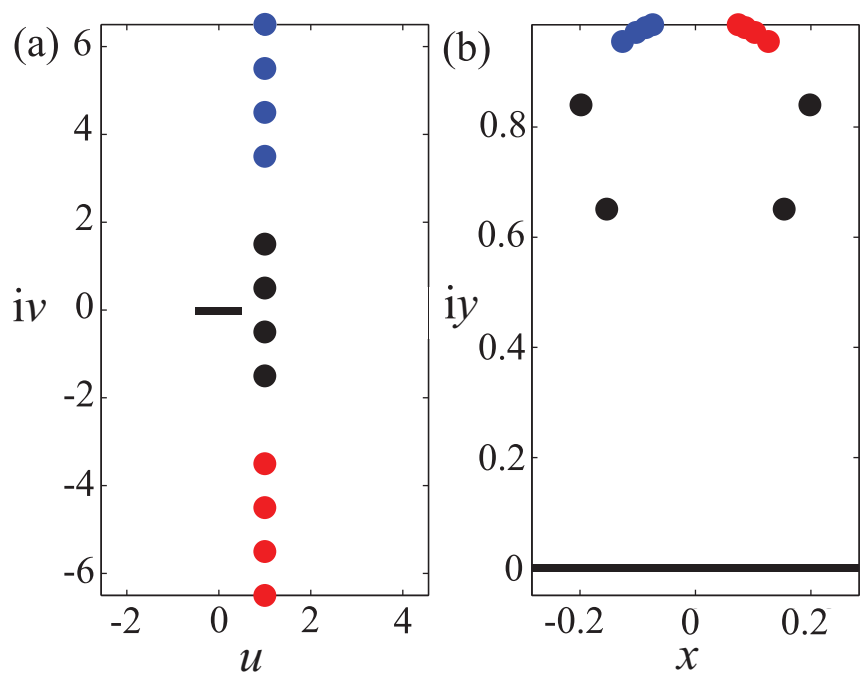

FIG. 2. (Color online) An example of conformal mapping. (a) The probe positions around an insulating line defect, which is indicated by the black line. (b) The corresponding upper half-plane solution where the $x$ axis is an infinite border. The situation on the right can be solved analytically as opposed to the left.

\section{NUMERICAL MODEL}

With more than one defect there is no simple analytical solution to the electrostatic problem. To calculate the effect of a large number of randomly positioned defects, we must turn to the finite element method (FEM). The numerical simulations were performed using COMSOL Multiphysics 4.4 with LiveLink for Matlab. The sample was modeled as a two-dimensional square-shaped area and the four probe pins were introduced as points placed on a straight line in the center of the square along the $x$ direction and separated by the probe pitch $s$. One pin was modeled as a current source $\delta$ function and one as a current drain $\delta$ function, and the two remaining pins were used to monitor the electrostatic potential difference resulting from the current flow. Using point source currents in the calculations is a good approximation when considering the length scale of the probe pitch, which is in micrometers, compared to the physical contact size of $\sim 10 \mathrm{~nm}$. Defects were introduced as straight insulating lines, as were the boundaries of the sample, so that in both cases $\mathbf{J}_{s} \cdot \mathbf{n}=0$. Adaptive mesh refinement was used on an initial extra-coarse mesh with a maximum of two mesh refinement steps. With these mesh settings the result was within $1 \%$ of the fully converged solution even for the highest defect densities.

For numerical simulations, a side length of $10 s$ was chosen to reduce computational time for systems with a large number of defects. Due to the proximity of the sample edges, the resistance ratio of the down-scaled system, without added defects, has the value 1.20. To achieve a given defect density, the corresponding amount of defect center coordinates were homogeneously distributed in a square grid, across the sample. Each defect center was then given a random offset in the $x$ and $y$ directions and the offset amplitudes were at most half the distance between two grid points. In addition, each defect was given a random orientation. 


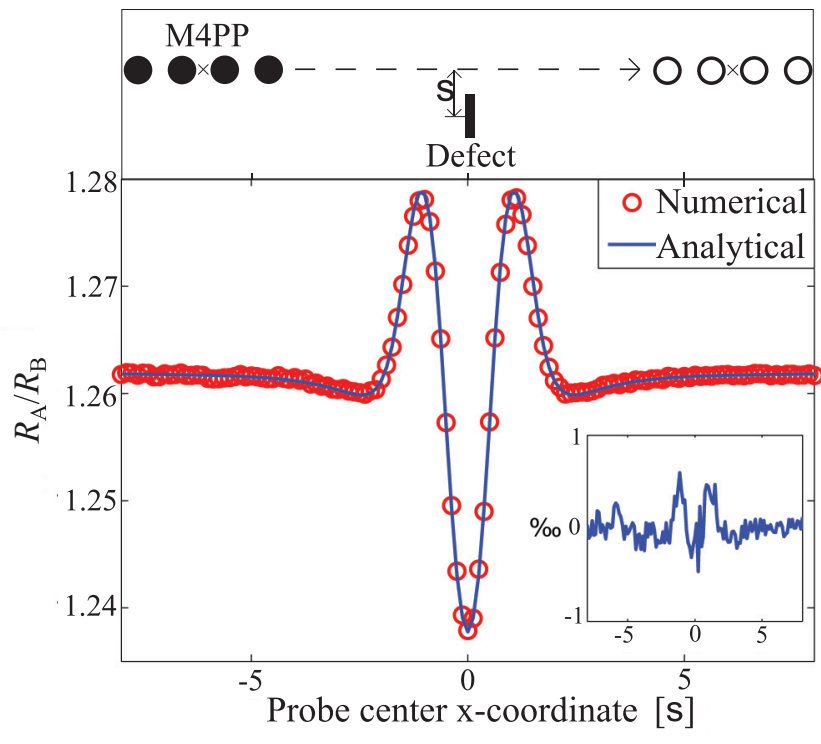

FIG. 3. (Color online) Analytically and numerically calculated resistance ratios for a probe scanned past a single line defect of length $s$, as indicated by the sketch in the top inset. The lower right inset shows the relative difference between the two models.

The validity of the FEM model was verified by setting up calculations where a probe was scanned past single defects in various configurations relative to the probe axis and scan direction. In all cases the calculated probe response was compared to the analytical result from conformal mapping. An example is shown in Fig. 3, where the top inset shows the probe-defect configuration. The probe was aligned and scanned along the $x$ direction, while the defect of length $s$ was aligned along the $y$ direction and displaced the distance $s$ in the $y$ direction from the probe scan axis. The resulting resistance ratios from numerical and analytical calculations are compared in Fig. 3. To numerically reproduce the resistance ratio of the defect-free semi-infinite sheet, $R_{\mathrm{A}} / R_{\mathrm{B}}=1.262$, we found that the sample side length had to be at least $450 \mathrm{~s}$. For this reason, the sample used in the numerical model for this verification was $450 s \times 450 s$. The difference between the numerical and the analytical result was below $0.1 \%$, as shown in the lower right inset in Fig. 3, which is evidence of an excellent agreement between the FEM model and the analytical result and thus serves to verify the FEM model.

\section{SENSITIVITY ANALYSIS}

In this work it is essential to visualize and clarify the transition from 2D to 1D-like transport. The signature of 1D transport appears when resistances measured in two different pin configurations, A and B, become equal. For measurements in two different pin configurations the resulting sheet current densities must differ even when the measured resistances are identical; thus the sheet current densities alone do not clearly reveal the transport dimensionality. See Supplemental Material for sheet current densities of 1D-like transport [18]. The sensitivity as explained below will, however, clearly illustrate the dimensionality of the transport.
The sensitivity of an M4PP measurement is a very useful concept that reveals detailed insight into which part of a sample contributes to the measured transfer resistance $R_{T}$. Here we use a dimensionless sensitivity $S_{T}$ defined [13] as $S_{T}=s^{2} \delta R_{T} /\left(\delta R_{0} \delta \Omega\right)$, where $\delta R_{0}$ is a small local deviation in sheet resistance $R_{0}$ within the incremental area $\delta \Omega$, and $\delta R_{T}$ is the resulting change in measured transfer resistance. In the Appendix we show that the sensitivities of $\mathrm{A}$ and $\mathrm{B}$ configuration measurements are

$$
S_{\mathrm{A}}=s^{2} \frac{\mathbf{J}_{\mathrm{A}} \cdot \mathbf{J}_{\mathrm{A}^{\prime}}}{I I^{\prime}} \quad \text { and } \quad S_{\mathrm{B}}=s^{2} \frac{\mathbf{J}_{\mathrm{B}} \cdot \mathbf{J}_{\mathrm{B}^{\prime}}}{I I^{\prime}},
$$

respectively [13]. Here $\mathbf{J}_{\mathrm{A}}, \mathbf{J}_{\mathrm{A}^{\prime}}, \mathbf{J}_{\mathrm{B}}$, and $\mathbf{J}_{\mathrm{B}^{\prime}}$ are the local sheet current densities in the respective measurement configurations, while $I$ and $I^{\prime}$ are the total measurement currents used in the measurements. Multiplication by the probe pitch squared renders the sensitivity dimensionless and eases comparison of absolute sensitivity values for different samples. Specifically, the sensitivities in a defective sample can be calculated to reveal details of how defects alter the measurements. By using the definition of the sensitivity we have for the resistance difference $R_{\mathrm{A}}-R_{\mathrm{B}}=\int_{\Omega} R_{0}\left(S_{\mathrm{A}}-S_{\mathrm{B}}\right) d \Omega / s^{2}$, and clearly in cases of identical sensitivities the resistances become identical.

\section{1D MEASUREMENTS}

In recent work we found that the numerical model qualitatively reproduces both the 2D and 1D current transport behaviors [8], which were earlier found experimentally [7]. To investigate the cause of the two dominant measurement signatures we consider two representative cases and use sensitivity analysis on their simulated sheet current densities. Sensitivity plots of $S_{\mathrm{A}}$ and $S_{\mathrm{B}}$ and their difference $S_{\mathrm{A}}-S_{\mathrm{B}}$ for the two typical simulations are shown in Fig. 4, where the white dots indicate the probe pins and the black lines are insulating defects. Figures 4(a)-4(c) (left column) are for a system containing a defect density of $1 s^{-2}$ with $R_{\mathrm{A}} / R_{\mathrm{B}}=1.205$, corresponding to that of a sample with the current limited only by the finite area sample boundary, and thus the figures exhibit 2D-like current transport characteristics. For comparison, the three inset images show the respective sensitivities for pure 2D current transport.

Figures 4(d)-4(f) (right column) are for a system containing a defect density of $4.84 s^{-2}$ and $R_{\mathrm{A}} / R_{\mathrm{B}}=1$, corresponding to 1D-like current transport characteristics. Here a larger fraction of the sheet area is characterized by having a higher value of sensitivity than in the left column. This can be explained by the large number of defects obstructing the path of least resistance, which forces the current on a longer route.

In the 2D-like case (left column) the difference in sensitivity between the two configurations is clearly visible in Fig. 4(c). This explains why different resistances are measured in the two configurations, yielding $R_{\mathrm{A}} / R_{\mathrm{B}}=1.205$. For the $1 \mathrm{D}$-like case (right column) the difference in sensitivity is mapped in Fig. 4(f). The largest values found here are on the order of $10^{-3}$ and thus very small compared to the 2D-like case. This shows that the areas that contribute to the measured resistances are essentially identical, and therefore identical resistances were measured, and $R_{\mathrm{A}} / R_{\mathrm{B}}=1$ results. In this 


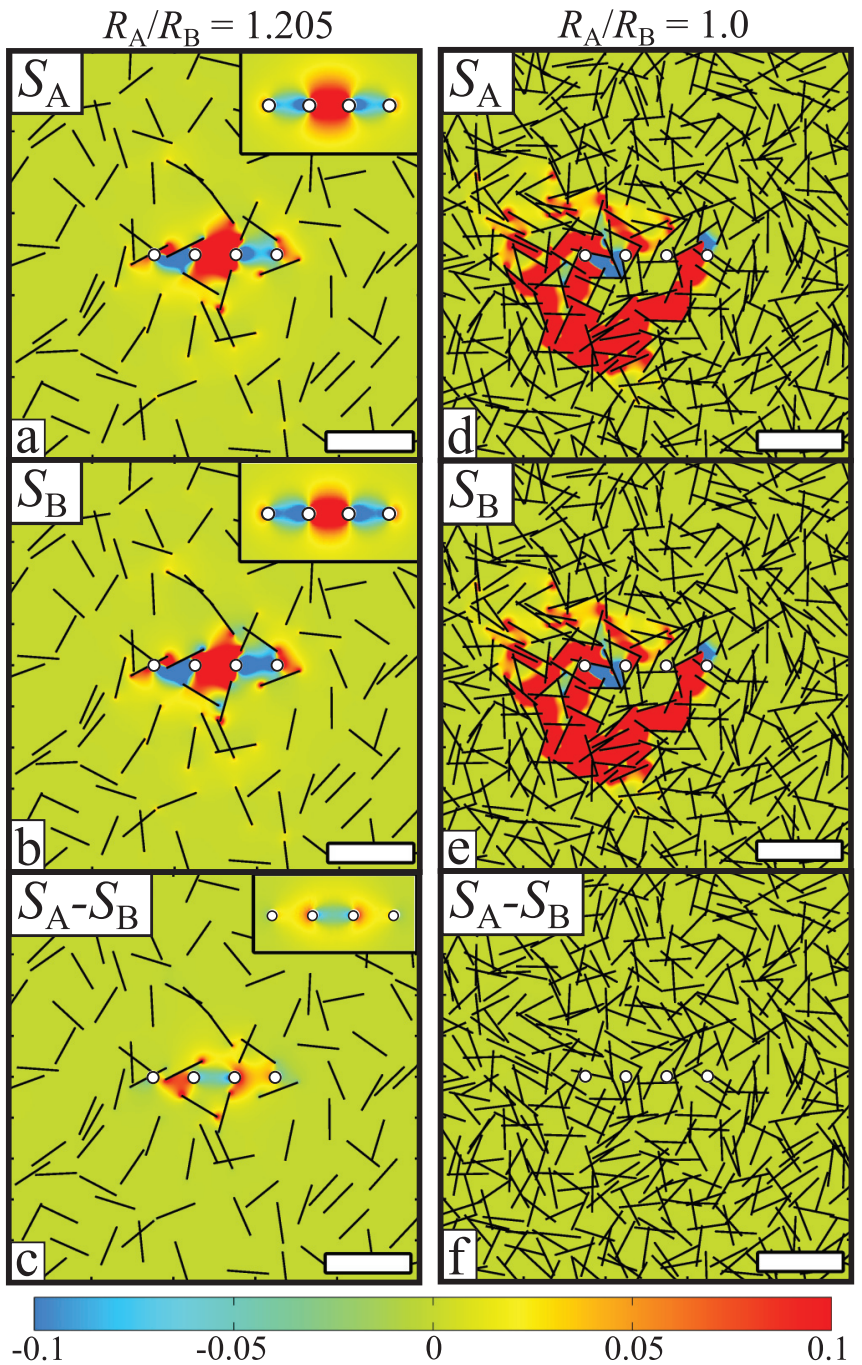

FIG. 4. (Color online) Sensitivity maps for typical 2D and 1D signature measurements where the four white dots represent the probe pin positions, and the black lines are insulating line defects. (a) and (b) are the $\mathrm{A}$ and $\mathrm{B}$ configuration sensitivity maps for a $2 \mathrm{D}$ signature case. (c) is the difference between the values $S_{\mathrm{A}}$ and $S_{\mathrm{B}}$ from (a) and (b). The inset images are the corresponding sensitivity maps for the zero-defect case. (d) and (e) are the A and B configuration sensitivity maps for the 1D signature case. (f) is the difference between the values $S_{\mathrm{A}}$ and $S_{\mathrm{B}}$ from (d) and (e), and is on the order of $10^{-3}$, which is very small compared to (c). The scale bar is $2 s$ ( $s$ is the probe pitch).

case $R_{\mathrm{A}}-R_{\mathrm{B}}=\int_{\Omega} R_{0}\left(S_{\mathrm{A}}-S_{\mathrm{B}}\right) d \Omega / s^{2}=0$ and thus identical resistances are measured.

\section{CONCLUSION}

We have shown that sensitivity analysis of M4PP measurements on 2D materials with extended defects gives considerable insight into the macroscopic transport properties of the materials. In particular we have shown that the sensitivities $S_{\mathrm{A}}$ and $S_{\mathrm{B}}$ in the two M4PP configurations A and B change dramatically when the defect density is increased. At low defect densities, $S_{\mathrm{A}}$ and $S_{\mathrm{B}}$ differ significantly and are localized to an area in the vicinity of the probe pins. As a result the measured resistances $R_{\mathrm{A}}$ and $R_{\mathrm{B}}$ differ, which is characteristic of $2 \mathrm{D}$ transport; the resistance ratio $R_{\mathrm{A}} / R_{\mathrm{B}}$ then becomes $\ln 4 / \ln 3$ for an infinite sample. In contrast, at high defect densities $S_{\mathrm{A}}$ and $S_{\mathrm{B}}$ become essentially identical and localized to a low dimensional path between the probe pins. As a result, the measured resistances $R_{\mathrm{A}}$ and $R_{\mathrm{B}}$ become identical (exactly the same part of the sample is measured), with the ratio $R_{\mathrm{A}} / R_{\mathrm{B}}=1.0$, a clear $1 \mathrm{D}$ signature. This analysis explains the similar behavior observed experimentally on defective graphene in Ref. [7]. The sensitivities were calculated using a FEM model, which was verified by comparison to an analytical calculation for the single-defect case, which we solved exactly by use of conformal mapping.

\section{ACKNOWLEDGMENTS}

Center for Individual Nanoparticle Functionality (CINF, DNRF54) and Center for Nanostructured Graphene (CNG, DNRF58) are funded by the Danish National Research Foundation. This work was supported by the Villum Foundation, Project No. VKR023117.

\section{APPENDIX}

For the derivation of the sensitivities, Eq. (2), we use an approach similar to that of Paul and Cornils [12]. We now consider a $2 \mathrm{D}$ region $\Omega$ with an insulating boundary $\omega$ (shown in Fig. 5) such that the sheet current density $\mathbf{J}_{s} \cdot \mathbf{n}=0$ on $\omega$, except at four electrodes (like the four pins in an M4PP) where a current $I_{i}$ may flow out of the electrode $i \in[1,2,3,4]$ with the potential $V_{i}$. The sheet current densities are considered divergence free, which means that we can write

$$
\nabla \cdot\left(\Phi \tilde{\mathbf{J}}_{s}\right)=\nabla \Phi \cdot \tilde{\mathbf{J}}_{s}+\Phi \nabla \cdot \tilde{\mathbf{J}}_{s}=\nabla \Phi \cdot \tilde{\mathbf{J}}_{s}=-\mathbf{E} \cdot \tilde{\mathbf{J}}_{s},
$$

where $\Phi$ is the potential in region $\Omega, \mathbf{E}=-\nabla \Phi$ the electric field, and $\tilde{\mathbf{J}}_{s}$ is the sheet current density in another region $\tilde{\Omega}$. Taking an integral over the region $\Omega$, and applying Stokes theorem, we get the following identity:

$$
\int_{\Omega} \nabla \cdot\left(\Phi \tilde{\mathbf{J}}_{s}\right) d \Omega=\int_{\omega} \Phi \tilde{\mathbf{J}}_{s} \cdot \mathbf{n} d \omega=-\int_{\Omega} \mathbf{E} \cdot \tilde{\mathbf{J}}_{s} d \Omega .
$$

The boundary integral is easily evaluated since the boundary conditions make the integral vanish except at the electrodes,

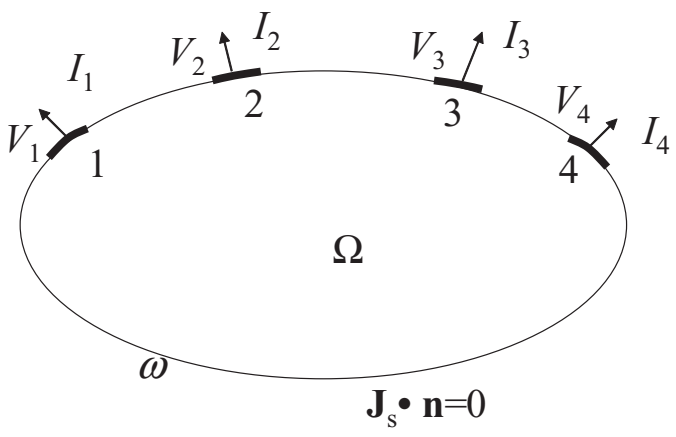

FIG. 5. Illustration of a $2 \mathrm{D}$ region $\Omega$ with insulating boundary except for four perfect contacts, Nos. 1-4. 
and thus a sum results:

$$
\int_{\omega} \Phi \tilde{\mathbf{J}}_{s} \cdot \mathbf{n} d \omega=\sum_{i=1}^{4} V_{i} \tilde{I}_{i}
$$

If we now consider the two regions identical but corresponding to reciprocal configurations, e.g., $\mathrm{A}$ and $\mathrm{A}^{\prime}$, only two terms remain in the sum, and from Eqs. (A2) and (A3) we find the relation

$$
V_{k} \tilde{I}_{k}+V_{\ell} \tilde{I}_{\ell}=-\left(V_{k}-V_{\ell}\right) \tilde{I}_{k \ell}=-\int_{\Omega} \mathbf{E} \cdot \tilde{\mathbf{J}}_{s} d \Omega,
$$

where $i, j, k, \ell \in[1,2,3,4]$ and $i \neq j \neq k \neq \ell$. From this result the transfer resistance $R_{T}=\left(V_{k}-V_{\ell}\right) / I_{i j}$ becomes

$$
R_{T}=\frac{V_{k}-V_{\ell}}{I_{i j}}=\frac{1}{I_{i j} \tilde{I}_{k \ell}} \int_{\Omega} \mathbf{E} \cdot \tilde{\mathbf{J}}_{s} d \Omega .
$$

At zero magnetic field the electric field and sheet current density are related as $\mathbf{E}=R_{0} \mathbf{J}_{s}$, where $R_{0}$ is the sheet resistance, and thus a general expression for the transfer resistance as a function of the current densities in the two configurations becomes

$$
R_{T}=\tilde{R}_{T}=\frac{1}{I \tilde{I}} \int_{\Omega} R_{0} \mathbf{J}_{s} \cdot \tilde{\mathbf{J}}_{s} d \Omega .
$$

Now we can define sensitivity as the change in transfer resistance relative to a change in local direct sheet resistance $\left(\delta R_{0}\right)$ in a small region $\delta \Omega$ as

$$
S=s^{2} \frac{\delta R_{T}}{\delta R_{0} \delta \Omega}=s^{2} \frac{\mathbf{J}_{s} \cdot \tilde{\mathbf{J}}_{s}}{I \tilde{I}},
$$

where the sensitivity has been made dimensionless by multiplication of $s^{2}$. This result was also given in Ref. [13] without proof.
[1] F. Banhart, J. Kotakoski, and A. V. Krasheninnikov, ACS Nano 5, 26 (2011).

[2] A. W. Tsen, L. Brown, M. P. Levendorf, F. Ghahari, P. Y. Huang, R. W. Havener, C. S. Ruiz-Vargas, D. A. Muller, P. Kim, and J. Park, Science 336, 1143 (2012).

[3] P. Y. Huang, C. S. Ruiz-Vargas, A. M. van der Zande, W. S. Whitney, M. P. Levendorf, J. W. Kevek, S. Garg, J. S. Alden, C. J. Hustedt, Y. Zhu, J. Park, P. L. McEuen, and D. A. Muller, Nature 469, 389 (2011).

[4] S. Chen, Q. Li, Q. Zhang, Y. Qu, H. Ji, R. S. Ruoff, and W. Cai, Nanotechnology 23, 365701 (2012).

[5] W. Zhu, T. Low, V. Perebeinos, A. A. Bol, Y. Zhu, H. Yan, J. Tersoff, and P. Avouris, Nano Lett. 12, 3431 (2012).

[6] J.-H. Chen, C. Jang, S. Xiao, M. Ishigami, and M. S. Fuhrer, Nat. Nanotechnol. 3, 206 (2008).

[7] J. C. D. Buron, D. H. Petersen, P. Bøggild, D. G. Cooke, M. Hilke, J. Sun, E. Whiteway, P. F. Nielsen, O. Hansen, A. Yurgens, and P. U. Jepsen, Nano Lett. 12, 5074 (2012).

[8] M. R. Lotz, M. Boll, O. Hansen, D. Kjær, P. Bøggild, and D. H. Petersen, Appl. Phys. Lett. 105, 053115 (2014).
[9] J. D. Buron, F. Pizzocchero, B. S. Jessen, T. J. Booth, P. F. Nielsen, O. Hansen, M. Hilke, E. Whiteway, P. U. Jepsen, P. Bøggild, and D. H. Petersen, Nano Lett. 14, 6348 (2014).

[10] D. W. Koon and C. J. Knickerbocker, Rev. Sci. Instrum. 63, 207 (1992).

[11] D. H. Petersen, R. Lin, T. M. Hansen, E. Rosseel, W. Vandervorst, C. Markvardsen, D. Kjær, and P. F. Nielsen, J. Vacuum Sci. Technol. B 26, 362 (2008).

[12] O. Paul and M. Cornils, Appl. Phys. Lett. 95, 232112 (2009).

[13] F. Wang, D. H. Petersen, T. M. Hansen, T. R. Henriksen, P. Bøggild, and O. Hansen, J. Vac. Sci. Technol. B 28, C1C34 (2010).

[14] D. Koon, F. Wang, D. Petersen, and O. Hansen, J. Appl. Phys. 114, 163710 (2013).

[15] D. W. Koon, Rev. Sci. Instrum. 77, 094703 (2006).

[16] D. W. Koon, F. Wang, D. H. Petersen, and O. Hansen, J. Appl. Phys. 116, 133706 (2014).

[17] D. H. Petersen, O. Hansen, R. Lin, and P. F. Nielsen, J. Appl. Phys. 104, 013710 (2008).

[18] See Supplemental Material at http://link.aps.org/supplemental/ 10.1103/PhysRevB.90.245432 for a comparison of sheet current densities and sensitivity of 1D-like transport. 